\title{
Introduction to Diffusive Representation
}

\author{
C. Casenave* G. Montseny* \\ * CNRS; LAAS; 7 avenue du colonel Roche, F-31077 Toulouse, France, \\ Université de Toulouse; UPS, INSA, INP, ISAE; LAAS; F-31077 \\ Toulouse, France (e-mail: casenave@laas.fr, montseny@laas.fr).
}

\begin{abstract}
Diffusive representation is an operator theory elaborated during the last years. It is devoted to time-nonlocal problems, allowing significant simplifications for analysis and numerical realization of integral time operators encountered in many physical situations. Namely, most of the shortcomings induced by time-nonlocal formulations are by-passed by use of suitable time-local state realizations deduced from diffusive representation and whose numerical approximations are straightforward, thanks to good properties of diffusion equations. In this paper, we introduce the notion of diffusive representation and its dual one: the diffusive symbol, and we briefly describe the associated mathematical framework and numerical techniques. The interest of this approach is highlighted by numerical examples.
\end{abstract}

Keywords: Causal operator, State-space realization, Distribution, Symbol, Convolution integral, Dynamic system, Distributed-parameter system.

\section{INTRODUCTION}

Linear causal integral operators, that is operators of the form:

$$
\mathcal{H}: u \mapsto\left(t \mapsto(\mathcal{H} u)(t)=\int_{-\infty}^{t} \mathbf{h}(t, s) u(s) d s\right),
$$

are often present in models of physics. So it is important to study them even if the task is tricky, especially because the class of considered operators is large. Among others, it contains rational operators and, when the kernel $\mathbf{h}$ is possibly a distribution, the differential operators.

For analysis and also numerical implementation purposes, it can be judicious to build new formulations of such operators, namely state realizations which have the interesting property to involve time-local expressions only, in opposite to the integral formulation (1) which is hereditary in the sense that the value of $\mathcal{H} u$ at a time $t$ explicitly depends on all the past of $u$. For example, classical Padé approximations are devoted to this problem.

The so-called diffusive representation (Montseny [2005]) is a theory (the beginnings of which can be found for example in Montseny [1991], Montseny et al. [1993b,a], Staffans [1994]) devoted to exact as well as approximate state realizations of a wide class of integral operators $\mathcal{H}$, that is, when the support of $u$ is in $\mathbb{R}^{+}$, to some formulations of $\mathcal{H} u$ of the following form:

$$
\begin{aligned}
\left(\partial_{t}-A\right) X & =B u, X(0)=0, \\
\mathcal{H} u & =C X,
\end{aligned}
$$

with $A, B$ and $C$ some suitable time-local operators. From the numerical point of view, thanks to the locality of the involved operators, a "step by step" integration (which does not need the memorization of the past of $u$ ) can be implemented. In fact, the past of $u$ is summarized in the "state-variable" $X$ used in this "synthesis" of $\mathcal{H}$. Note that contrarily to standard methods devoted to approximation of nonrational operators $\mathcal{H}$, in the diffusive representation approach, the main object the analysis focuses on is a suitable time-frequency transformation of the input $u$ (namely the " $\gamma$-representation" of $u$, which will be denoted by $\psi)$.

By means of diffusive representation, we get some various state realizations which make most of time analysis and simulation of complex systems much more simple than under the standard integral formulation. This methodology is of general scope because it offers a unified and useful mathematical framework in which first the standard algebraic operations about integral operators are well defined, and second, efficient numerical approximations can be easily built. According to diffusive representation, simple rational operators as well as complex non-convolution ones are represented in the same way.

The main notions and some recent results relating to this theory are presented in this paper in a simplified way. A complete theoretical statement will be found in Montseny [2005] and various applications to non trivial problems will be found in Audounet and Roquejoffre [1998], Carmona and Coutin [1998a,b], Carmona et al. [2003], Casenave and Montseny [2009, 2008], Degerli et al. [Ap. 1999], Garcia and Bernussou [1998], Lenczner and Montseny [2005], Lenczner et al. [2010], Levadoux and Montseny [2003], Montseny [2007], Mouyon and Imbert [2002], Rumeau et al. [2006], Bidan et al. [2001], Audounet et al. [1998], Degerli et al. [1999], Devy-Vareta and Montseny [2001], Helie and Matignon [2006], Laudebat et al. [2004], Lavernhe et al. [2001], Lavernhe and Solhusvik [1998], Leger and Pontier [1999], Lubich and Schädler [2002], Matignon [July 2001], Matignon and Prieur [2005], Mbodge et al. [1994], Mbodge and Montseny [1995], Montseny [2002b,a, 1998], Montseny et al. [2000], Nihtila and Tervo [2002], Rouzaud [1998]. 


\section{GENERAL PRINCIPLE}

We denote by $\mathcal{L}$ the Laplace transform defined by: $(\mathcal{L} f)(p)=\int_{0}^{+\infty} e^{-p t} f(t) d t$.

Given $h \in L_{\text {loc }}^{1}\left(\mathbb{R}^{+}\right)$such that $h(t) \underset{t \rightarrow+\infty}{\rightarrow} 0$, we consider a linear causal convolution operator defined, on any continuous function $u: \mathbb{R}^{+} \rightarrow \mathbb{R}$, by:

$$
u \mapsto\left(t \mapsto \int_{0}^{t} h(t-s) u(s) d s\right) .
$$

We denote by $H$ the Laplace transform of $h$ and $H\left(\partial_{t}\right)$ the convolution operator defined by (4).

Let $u^{t}(s)=\mathbf{1}_{]-\infty, t]}(s) u(s)$ be the restriction of $u$ to its past and $u_{t}(s)=u^{t}(t-s)$ the so-called "history" of $u$. From causality of $H\left(\partial_{t}\right)$, we deduce:

$$
\left[H\left(\partial_{t}\right)\left(u-u^{t}\right)\right](t)=0 \text { for all } t
$$

then, we have for any continuous function $u$ :

$$
\left[H\left(\partial_{t}\right) u\right](t)=\left[\mathcal{L}^{-1}(H \mathcal{L} u)\right](t)=\left[\mathcal{L}^{-1}\left(H \mathcal{L} u^{t}\right)\right](t) .
$$

We then define:

$$
\Psi_{u}(t, p):=\mathrm{e}^{p t}\left(\mathcal{L} u^{t}\right)(p)=\left(\mathcal{L} u_{t}\right)(-p) .
$$

By computing $\partial_{t} \mathcal{L} u_{t}$, Laplace inversion and use of (6), the following result can be proved:

Proposition 1. $\Psi_{u}$ is solution of the differential equation:

$$
\partial_{t} \Psi(t, p)=p \Psi(t, p)+u, \quad t>0, \quad \Psi(0, p)=0,
$$

and for any $b \geqslant 0$ :

$$
\left[H\left(\partial_{t}\right) u\right](t)=\frac{1}{2 i \pi} \int_{b-i \infty}^{b+i \infty} H(p) \Psi_{u}(t, p) d p .
$$

The main advantage of the "state formulation" $(8,9)$ is its time-locality, that we don't have with (4). However in return, equation (8) generates some heavy difficulties because $\operatorname{Re}(p) \geqslant 0$ on $] b-\mathrm{i} \infty, b+\mathrm{i} \infty[$, especially from the numerical approximation point of view. To suppress this problem, we introduce in the sequel a suitable complex arc $\gamma \subset \mathbb{R}^{-}$such that, under some hypothesis on $H\left(\partial_{t}\right)$ :

- $\left[H\left(\partial_{t}\right) u\right](t)=\frac{1}{2 i \pi} \int_{\gamma} H(p) \Psi_{u}(t, p) d p$,

- equation (8) is dissipative thanks to the property: $\operatorname{Re}(p)<0$ almost everywhere on $\gamma$.

\section{FUNDAMENTAL THEOREM}

Let $J \subseteq \mathbb{R}$ and $\gamma \in W^{1, \infty}(J ; \mathbb{C})^{1}$ defining a closed ${ }^{2}$ contour in $\mathbb{C}^{-}$(also denoted $\left.\gamma\right)$; we denote $\Omega_{\gamma}^{+}$the exterior domain defined by $\gamma$, and $\Omega_{\gamma}^{-}=\mathbb{C} \backslash \overline{\Omega_{\gamma}^{+}}$, such as shown in Fig. 1a.

We suppose there exists $\left.\alpha_{\gamma} \in\right] \frac{\pi}{2}, \pi[$ and $a \in \mathbb{R}$ such that:

- $e^{\mathrm{i}\left[-\alpha_{\gamma}, \alpha_{\gamma}\right]} \mathbb{R}_{+}+a \subset \Omega_{\gamma}^{+}$,

- $\mathbb{R}_{+}^{*}+\mathrm{i} \mathbb{R} \subset \Omega_{\gamma}^{+}$, with $\gamma \cap \mathrm{i} \mathbb{R}$ finite set.

We also suppose that there exists $b, c>0$ such that the function $\gamma$ satisfies the following property:

$$
b \leq\left|\gamma^{\prime}(\xi)\right| \leq c \quad \xi \text {-a.e. }
$$

Let $H:=\mathcal{L} h$ be the Laplace-symbol of the causal integral operator $\mathcal{H}$ with impulse response $h \in L_{\text {loc }}^{1}\left(\mathbb{R}^{+}\right)$. We then have:

$1 W^{1, \infty}(J ; \mathbb{C})$ is the classical Sobolev space of complex functions $f$ defined on $J$, such that $f, f^{\prime} \in L^{\infty}(J ; \mathbb{C})$.

2 Possibly at infinity

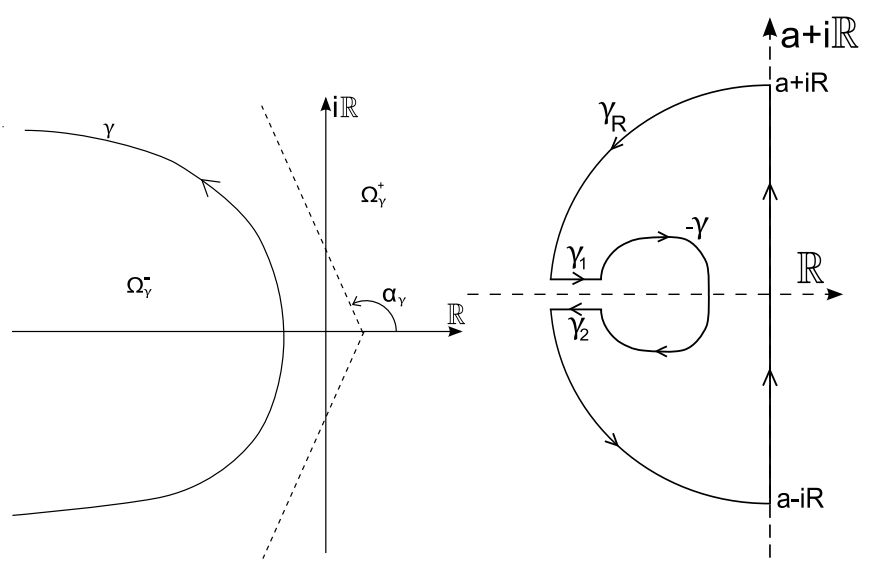

(a) Example of contour $\gamma$.

(b) Contour $\Gamma$.

Fig. 1. Contours $\gamma$ and $\Gamma$

Theorem 2. If:

(i) $H$ is holomorphic in $\Omega_{\gamma}^{+}$and the possible singularities on $\gamma$ are limited to a finite number of branching points denoted $p_{k}$ such that, with $c_{r}^{k}$ the circle of center $p_{k}$ and radius $r$ :

$$
\forall k, \forall t, \forall s>0, \quad \int_{c_{r}^{k}} e^{p s} H(p) d p \underset{r \rightarrow 0}{\longrightarrow} 0,
$$

(ii) the trace of $H$ on $\gamma$ is locally Lebesgue-integrable,

(iii) there exists a sequence $\rho_{n}$ such that $\rho_{n} \underset{n \rightarrow+\infty}{\longrightarrow}+\infty$ and $H\left(\rho_{n} e^{i \theta}\right) \underset{n \rightarrow+\infty}{\longrightarrow} 0$ uniformly with respect to $\theta \in\left[\frac{\pi}{2}, \frac{3 \pi}{2}\right]$,

then, for all $u$ with support in $\mathbb{R}^{+},(\mathcal{H} u)(t)=\left(H\left(\partial_{t}\right) u\right)(t)$ is expressed by:

$$
(\mathcal{H} u)(t)=\int_{J} \mu(\xi) \psi(t, \xi) d \xi=<\mu, \psi(t, .)>,
$$

where:

$$
\mu(\xi)=\frac{\gamma^{\prime}(\xi)}{2 \mathrm{i} \pi} H(\gamma(\xi)),
$$

and $\psi(t, \xi)$ is solution of:

$$
\partial_{t} \psi(t, \xi)=\gamma(\xi) \psi(t, \xi)+u(t), \xi \in \mathbb{R}, \psi(0, .)=0 .
$$

Proof 3. Assume that $\gamma$ is bounded ${ }^{3}$. We have, for all $t>0$, and $s>0$ :

$$
h(s)=\left(\mathcal{L}^{-1} H\right)(s)=\frac{1}{2 \mathrm{i} \pi} \lim _{R \rightarrow+\infty} \int_{a-i R}^{a+i R} e^{p s} H(p) d p,
$$

with $a>0$.

Let consider the contour $\Gamma$ as defined on Fig. $1 b^{4}$ :

$$
\Gamma=(a+i[-R, R]) \cup \gamma_{R} \cup \gamma_{1} \cup \gamma_{2} \cup(-\gamma),
$$

with $\gamma_{R}=\{z \in \mathbb{C} /|z|=R, \operatorname{Re}(z) \leq 0\}$ and $\gamma_{1}=-\gamma_{2}$. Then we have the following results:

- from hypothesis $(i), \int_{\Gamma} e^{p s} H(p) d p=0$,

- as $\gamma_{1}=-\gamma_{2}, \int_{\gamma_{1}} e^{p s} H(p) d p=-\int_{\gamma_{2}} e^{p s} H(p) d p$,

- from Jordan-lemma and hypothesis $(i),($ iii $)$ :

$$
\lim _{R \rightarrow+\infty} \int_{\gamma_{R}} e^{p s} H(p) d p=0 .
$$

3 The unbounded case can be treated in a similar way, up to technical adaptations.

4 The contour $\gamma$ being closed, there exists $R>0$ for which such a contour $\Gamma$ can be defined. 
So, for all $t>0, s>0$ :

$$
h(s)=\frac{1}{2 \mathrm{i} \pi} \int_{\gamma} e^{p s} H(p) d p=\int_{J} e^{\gamma(\xi) s} \mu(\xi) d \xi,
$$

with $\mu(\xi)=\frac{\gamma^{\prime}(\xi)}{2 \mathrm{i} \pi} H(\gamma(\xi))$, the integral on $J$ being a Lebesgue one thanks to hypothesis $(i i)$. For any causal locally integrable function $u$, and for all $t>0$ we so have:

$$
(h * u)(t)=\int_{0}^{t} \int_{J} e^{\gamma(\xi) s} \mu(\xi) d \xi u(t-s) d s .
$$

By assumption, we also have, for any causal locally integrable $u$ and for all $t>0,(h * u)(t)<+\infty$. So we can apply the Fubini theorem on space $L^{1}(0, t) \otimes L^{1}\left(\mathbb{R}_{\xi}\right)$; we get:

$$
\begin{aligned}
(h * u)(t) & =\int_{J} \mu(\xi) \int_{0}^{t} e^{\gamma(\xi) s} u(t-s) d s d \xi \\
& =\int_{J} \mu(\xi) \psi(t, \xi) d \xi,
\end{aligned}
$$

where $\psi(t, \xi)=\int_{0}^{t} e^{\gamma(\xi) s} u(t-s) d s$ is the (unique) solution of (16).

Remark 4. - From theorem 2, we get a state realization of the convolution operator $\mathcal{H}$. This realization is of infinite dimension because $\xi \in \mathbb{R}$. Nevertheless, thanks to the sectorial condition (10), equation (16) is of diffusive type (Yosida [1965]) and so, some approximations of reasonable finite dimension can be built.

- We denote $\mathcal{L}_{\gamma}$ the operator defined by:

$$
\left(\mathcal{L}_{\gamma} g\right)(\xi)=\int_{0}^{+\infty} e^{\gamma(\xi) s} g(s) d s=(\mathcal{L} g)(-\gamma(\xi)) ;
$$

the adjoint of $\mathcal{L}_{\gamma}$, denoted $\mathcal{L}_{\gamma}^{*}$, is defined by:

$$
\left(\mathcal{L}_{\gamma}^{*} f\right)(s)=\int_{J} e^{\gamma(\xi) s} f(\xi) d \xi \mathbf{1}_{\mathbb{R}_{*}^{+}}(s)
$$

and we have (see proof of theorem 2), for all $t>0$ :

$$
\begin{aligned}
h(s) & =\left(\mathcal{L}_{\gamma}^{*} \mu\right)(s), \\
\psi(t, \xi) & =\left(\mathcal{L}_{\gamma} u_{t}\right)(\xi) .
\end{aligned}
$$

- In the proof, it has been shown that, under the hypothesis of theorem 2, the function $\mu$ defined by (15) is solution of the equation (25). In fact, this equation admits several solutions.

- From $(14,15,16),\left(H\left(\partial_{t}\right) u\right)(t)$ can be expressed:

$$
H\left(\partial_{t}\right) u(t)=\frac{1}{2 \mathrm{i} \pi} \int_{\gamma} H(p) \Psi_{u}(t, p) d p,
$$

with $\Psi_{u}(t, p)$ defined by (7). In the sequel, we will give a sense to this integral in more general cases where the trace of $H$ on $\gamma$ is no more locally integrable but defines a singular distribution.

\section{TERMINOLOGY AND NOTATIONS}

We now introduce a few definitions and notations.

- Let $\mathcal{L}_{+}\left(L^{2}(\mathbb{R})\right)$ be the algebra of causal and continuous linear operators in $L^{2}(\mathbb{R})$. The linear operator $\left(\partial_{t}-p I\right)$ admits an inverse in $\mathcal{L}_{+}\left(L^{2}(\mathbb{R})\right)$ if $\operatorname{Re}(p)<0$ (Yosida [1965]). So we can define:

Definition 1. The diffusive representation operator, denote $\mathfrak{R d}$, is the operator function:

$$
\begin{aligned}
\mathfrak{R d}: \mathbb{R}^{-*}+i \mathbb{R} & \longrightarrow \mathcal{L}_{+}\left(L^{2}(\mathbb{R})\right) \\
p & \longmapsto \mathfrak{R d}_{p}=\left(\partial_{t}-p I\right)^{-1} .
\end{aligned}
$$

- $\mathfrak{R d} u: p \in \mathbb{C}^{-} \longmapsto \mathfrak{R d}_{p} u \in L^{2}\left(\mathbb{R}_{t} ; \mathbb{C}\right)$ is called the diffusive representation of $u$; if $\operatorname{supp} u \subset \mathbb{R}_{t}^{+}$, then $(\mathfrak{R d} u)(p)=\Psi_{u}(., p)$ is the (unique) solution of the differential equation:

$$
\partial_{t} \Psi(t, p)=p \Psi(t, p)+u(t), \Psi(0, p)=0 ;
$$

so, $\psi:=\mathfrak{R d}_{\gamma} u:=(\mathfrak{R d} \circ \gamma) u=\Psi_{u}(., \gamma)$ is the (unique) solution of the (infinite dimensional) Cauchy problem:

$$
\partial_{t} \psi(t, \xi)=\gamma(\xi) \psi(t, \xi)+u(t), \quad \xi \in J, \psi(0, \xi)=0 .
$$

From (26), we then have:

$$
\left(\mathfrak{R} \mathrm{d}_{\gamma} u\right)(t, \xi)=\left(\mathcal{L}_{\gamma} u_{t}(t, .)\right)(\xi) \quad \xi \text {-a.e. }
$$

The function $\psi=\mathfrak{R d}_{\gamma} u$ is called the $\gamma$-representation of $u$.

- Any solution $\mu$ of the equation $h=\mathcal{L}_{\gamma}^{*} \mu$ is called a $\gamma$-symbol of $H\left(\partial_{t}\right)$; the particular solution given by (15) is called the canonical $\gamma$-symbol.

- An operator is said $\gamma$-diffusive (in the strict sense) if it admits a $\gamma$-symbol with $\gamma$ verifying (10) and (11).

- An operator $H\left(\partial_{t}\right)$ such that, for all causal locally integrable function $u$ :

$$
H\left(\partial_{t}\right) u=<\mu, \mathfrak{R d} \mathrm{d}_{\gamma} u>
$$

is said $\gamma$-realizable (or realizable on $\gamma$ ).

- With $\mu_{H}$ a $\gamma$-symbol of $H\left(\partial_{t}\right)$, the expression $<\mu_{H}, \mathfrak{R} \mathrm{d}_{\gamma}>$ is called the $\gamma$-realization of $H\left(\partial_{t}\right)$.

\section{OPTIMAL TOPOLOGICAL FRAMEWORK}

The results of theorem 2 can be extended to a larger class of operators, providing that the associated $\gamma$-symbols are extended to distributions (so, the expression $\left\langle\mu, \mathfrak{R d}_{\gamma} u\right\rangle$ will refer to a topological duality product).

In the sequel, we introduce some topological spaces $\Delta_{\gamma}$ and $\Delta_{\gamma}^{\prime}$ such that for any $u, \psi=\mathfrak{R d}_{\gamma} u \in \Delta_{\gamma}$ and for any $\mu \in \Delta_{\gamma}^{\prime}$, we get:

$$
<\mu, \psi>_{\Delta_{\gamma}^{\prime}, \Delta_{\gamma}}=H\left(\partial_{t}\right) u,
$$

where $H\left(\partial_{t}\right)$ is a convolution operator defined by its $\gamma$ symbol $\mu$.

\subsection{The spaces $\mathcal{D}_{L_{P}^{\infty}}$}

Let $L_{P}^{\infty}(\mathbb{R})$ be the Lebesgue space defined by the norm:

$$
\|\varphi\|_{L_{P}^{\infty}}=\sup _{\xi \in \mathbb{R}} P(\xi)|\varphi(\xi)| \text { with } P(\xi):=\sqrt{1+\xi^{2}} .
$$

Then the space:

$$
\mathcal{D}_{L_{P}^{\infty}}(\mathbb{R})=\left\{\varphi \in C^{\infty}(\mathbb{R}), \forall n \in \mathbb{N}, \partial_{\xi}^{n} \varphi \in L_{P}^{\infty}(\mathbb{R})\right\},
$$

is a Fréchet space; its topology is defined by the countable set of semi-norms: $\|\varphi\|_{n}=\left\|\partial_{\xi}^{n} \varphi\right\|_{L_{P}^{\infty}}$.

\subsection{Spaces $\boldsymbol{\Delta}_{\gamma}$ and $\boldsymbol{\Delta}_{\gamma}^{\prime}$}

In this section, we suppose in addition that the function $\gamma: \mathbb{R} \rightarrow \mathbb{C}$ is smooth and unbounded (that is $J=\mathbb{R}$ ). Without loss of generality, we also suppose that there exists $\xi_{0}$ and $\lambda>0$ such that:

$$
\begin{aligned}
& \text { - } \operatorname{Re} \gamma(\xi) \leq-\lambda|\xi| \quad \forall|\xi| \geq \xi_{0}, \\
& \text { - } \forall n>0, \partial_{\xi}^{n} \gamma \text { is bounded. }
\end{aligned}
$$

Let consider the space $L_{r}^{\infty}(\mathbb{R})$ of functions of $L^{\infty}(\mathbb{R})$ with right bounded support. 
Proposition 5. $\forall v \in L_{r}^{\infty}\left(\mathbb{R}_{s}\right), \psi:=\mathcal{L}_{\gamma} v \in \mathcal{D}_{L_{P}^{\infty}}(\mathbb{R})$.

Definition 2. $\boldsymbol{\Delta}_{\gamma}$ is the completion of $\mathcal{L}_{\gamma}\left(L_{r}^{\infty}\left(\mathbb{R}_{s}\right)\right)$ in $\mathcal{D}_{L_{p}^{\infty}}(\mathbb{R})$; it is a strict subspace of $\mathcal{D}_{L_{p}^{\infty}}(\mathbb{R})$.

Set of $v$ associated with $\boldsymbol{\Delta}_{\gamma} \quad$ Because $\mathcal{L}_{\gamma}$ is a regularizing operator, the $v$ such that $\mathcal{L}_{\gamma} v \in \boldsymbol{\Delta}_{\gamma}$ can be distributions. More precisely, it can be shown that for all $u \in \mathcal{D}^{\prime}\left(\mathbb{R}^{+}\right)$:

$$
\psi:=\mathcal{L}_{\gamma} u_{t} \in \boldsymbol{\Delta}_{\gamma}
$$

so the set of $u$ we can consider is sufficiently large for practical needs.

Definition 3. The space $\boldsymbol{\Delta}_{\gamma}^{\prime}$ is the topological dual of $\boldsymbol{\Delta}_{\gamma}$.

As usual, two convergence modes can be defined on $\boldsymbol{\Delta}_{\gamma}^{\prime}$ :

- the weak-* convergence:

$$
\mu_{n} \stackrel{*}{\rightarrow} 0 \Leftrightarrow \forall \psi \in \Delta_{\gamma},<\mu_{n}, \psi>\rightarrow 0 .
$$

- the strong convergence:

$$
\mu_{n} \rightarrow 0 \Leftrightarrow\left\{\begin{array}{l}
<\mu_{n}, \psi>\rightarrow 0 \text { uniformly } \\
\text { on any bounded set of } \boldsymbol{\Delta}_{\gamma} .
\end{array}\right.
$$

From definition 2 and because $\boldsymbol{\Delta}_{\gamma}$ is a strict subspace of $\mathcal{D}_{L_{P}^{\infty}}, \boldsymbol{\Delta}_{\gamma}^{\prime}$ is a quotient space of distributions:

Proposition 6. $\boldsymbol{\Delta}_{\gamma}^{\prime}=\mathcal{D}_{L_{p}^{\infty}}^{\prime} / \operatorname{ker} \mathcal{L}_{\gamma}^{*}$.

So $\boldsymbol{\Delta}_{\gamma}^{\prime}$ is constituted of equivalence classes (of $\gamma$-symbols), associated with the equivalence relation:

$$
\forall \mu_{1}, \mu_{2} \in \mathcal{D}_{L_{p}^{\infty}}^{\prime}, \mu_{1} \stackrel{\gamma}{\sim} \mu_{2} \Longleftrightarrow \mu_{1}-\mu_{2} \in \operatorname{ker} \mathcal{L}_{\gamma}^{*}
$$

Remark \%. $\mathcal{D}_{L_{p}^{\infty}}^{\prime} \subset \mathcal{D}^{\prime}$ : in general, the $\gamma$-symbols are distributions.

Class of operators with $\gamma$-symbols in $\boldsymbol{\Delta}_{\gamma}^{\prime}$

Proposition 8. (Montseny [2005]) Let $\mu \in \boldsymbol{\Delta}_{\gamma}^{\prime}$. The operator

$$
u \longmapsto<\mu, \mathfrak{R d _ { \gamma }} u>_{\boldsymbol{\Delta}_{\gamma}^{\prime}, \boldsymbol{\Delta}_{\gamma}}
$$

is the convolution operator with impulse response:

$$
h(t)=<\mu, e^{\gamma(.) t}>_{\boldsymbol{\Delta}_{\gamma}^{\prime}, \boldsymbol{\Delta}_{\gamma}}=\left(\mathcal{L}_{\gamma}^{*} \mu\right)(t) ;
$$

its Laplace-symbol is defined, for all $p \in \Omega_{\gamma}^{+}$by:

$$
H(p)=<\mu, \frac{1}{p-\gamma(.)}>_{\boldsymbol{\Delta}_{\gamma}^{\prime}, \boldsymbol{\Delta}_{\gamma}}=\left(\mathcal{L}_{\mathcal{L}_{\gamma}^{*}} \mu\right)(p) .
$$

The function $H$ verifies the properties:

- $H$ is holomorphic in $\Omega_{\gamma}^{+}$,

- $H(p) \rightarrow 0$ when $|p| \rightarrow+\infty$ in $\Omega_{\gamma}^{+}$.

Remark 9. The conditions $(44,45)$ are only necessary ones but in practice, they are also almost sufficient.

Proposition 10. Let $\mu \in \boldsymbol{\Delta}_{\gamma}^{\prime}$ and $u \in L_{\mathrm{loc}}^{\infty}\left(\mathbb{R}^{+}\right)$. Then, for any contour $\widetilde{\gamma}$ in $\Omega_{\gamma}^{+}$such that $\gamma \subset \Omega_{\gamma}^{-}$, we have:

$$
<\mu, \mathfrak{R d _ { \gamma }} u>_{\boldsymbol{\Delta}_{\gamma}^{\prime}, \boldsymbol{\Delta}_{\gamma}}=\frac{1}{2 \mathrm{i} \pi} \int_{\widetilde{\gamma}} H \Psi_{u} d p,
$$

where $H=\mathcal{L} \mathcal{L}_{\gamma}^{*} \mu$ and $\Psi_{u}(t,$.$) is the analytic continuation$ of $\Re \mathrm{d}_{\gamma} u$.

As $\frac{1}{2 \mathrm{i} \pi} \int_{\widetilde{\gamma}} H \Psi_{u} d p$ takes the same value whatever the contour $\widetilde{\gamma}$ in $\Omega_{\gamma}^{+}$, we can denote it $\frac{1}{2 \mathrm{i} \pi} \int_{\gamma} H \Psi_{u} d p$ (even if $H$ is not locally integrable on $\gamma$ ). In that sense, this extends formula (27).
Remark 11. The relation (46) is not true for all $<\mu, \psi>_{\boldsymbol{\Delta}_{\gamma}^{\prime}, \boldsymbol{\Delta}_{\gamma}}$ with $\psi \in \boldsymbol{\Delta}_{\gamma}$. Indeed, the elements $\psi$ of $\boldsymbol{\Delta}_{\gamma}$ being of $C^{\infty}$ class, they are not necessary the trace on $\gamma$ of a function analytic in the neighborhood of $\gamma$. So it is possible that $\Omega_{\gamma}^{+} \cap \Omega_{\gamma}^{-}$is not included in the intersection of analyticity domains of $H$ and $\Psi(t,$.$) .$

Canonical $\gamma$-symbols of $\boldsymbol{\Delta}_{\gamma}^{\prime}$ In each equivalence class, there is a unique canonical $\gamma$-symbol whose definition is given here after:

Definition 4. Let $\mu \in \boldsymbol{\Delta}_{\gamma}^{\prime}$ be an equivalence class of $\gamma$ symbols. The $\gamma$-symbol $\mu_{\mathrm{c}}$ defined by:

$$
\mu_{c}:=\frac{\gamma^{\prime}}{2 \mathrm{i} \pi}\left(\mathcal{L} \mathcal{L}_{\gamma}^{*} \mu\right)_{\mid \gamma^{+}} \in \boldsymbol{\Delta}_{\gamma}^{\prime}
$$

where (.) $\mid \gamma^{+}$is the right trace on $\gamma$ in the sense of distributions, is called the canonical $\gamma$-symbol of $\mu$.

The product $\sharp_{\gamma}$ in $\boldsymbol{\Delta}_{\gamma}^{\prime} \quad$ The composition product of operators has an equivalent in $\boldsymbol{\Delta}_{\gamma}^{\prime}$, denoted $\sharp_{\gamma}$ : if $\mu$ and $\nu$ are respective (class of) $\gamma$-symbols of $H\left(\partial_{t}\right)$ and $K\left(\partial_{t}\right)$, then $\mu \sharp_{\gamma} \nu$ is the (class of) $\gamma$-symbol(s) of $H\left(\partial_{t}\right) \circ K\left(\partial_{t}\right)$. Definition 5. Let $\mu, \nu \in \boldsymbol{\Delta}_{\gamma}^{\prime}$. The (class of) $\gamma$-symbol(s) denoted $\mu \sharp_{\gamma} \nu$ is characterized by the relation:

$$
\mathcal{L}_{\gamma}^{*}\left(\mu \sharp_{\gamma} \nu\right)=\left(\mathcal{L}_{\gamma}^{*} \mu\right) *\left(\mathcal{L}_{\gamma}^{*} \nu\right) .
$$

Proposition 12. The product $\sharp_{\gamma}$ is inner (and commutative) in $\boldsymbol{\Delta}_{\gamma}^{\prime}$ and separately sequentially continuous for the weak-* topology, that is:

$$
\left(<\nu_{n}, \psi>\underset{n \rightarrow \infty}{\stackrel{*}{\rightarrow}} 0\right) \Longrightarrow\left(<\mu \sharp_{\gamma} \nu_{n}, \psi>\underset{n \rightarrow \infty}{\stackrel{*}{\rightarrow}} 0\right) \text {. }
$$

\subsection{Spaces $\Delta_{\gamma}$ and $\Delta_{\gamma}^{\prime}$}

The duality spaces $\boldsymbol{\Delta}_{\gamma}$ and $\boldsymbol{\Delta}_{\gamma}^{\prime}$ are not the optimal framework for the diffusive representation. Among others things, the product $\sharp_{\gamma}$ is not continuous, the relation (46) is not true for all $\langle\mu, \psi\rangle$ with $\psi \in \boldsymbol{\Delta}_{\gamma}$ (see remark 11) and the contour $\gamma$ is necessarily smooth, which is frequently a shortcoming in practical situations.

So new topological spaces $\Delta_{\gamma}$ and $\Delta_{\gamma}^{\prime}$ have been introduced such that:

- $\forall \mu \in \Delta_{\gamma}^{\prime}, H($.$) is holomorphic in \Omega_{\gamma}^{+}$,

- $\forall \psi \in \Delta_{\gamma}, \Psi(t,$.$) is holomorphic in \Omega_{\gamma_{0}}^{-}$,

with $\widetilde{\gamma}_{0} \subset \Omega_{\gamma}^{+}$(see Fig. 2). Thus, the integral $\int_{\tilde{\gamma}} H_{\mu} \Psi_{u} d p$ will be well-defined on any contour $\widetilde{\gamma}$ in the intersection of the analyticity domains of $H_{\mu}$ and $\Psi_{u}$ (see Fig. 2) and will take the same value that will be denoted $\int_{\gamma} H_{\mu} \Psi_{u} d p$, by extension of formula (27).

The construction of the space $\Delta_{\gamma}$ (with topological dual $\left.\Delta_{\gamma}^{\prime}\right)$ is not described here: more details will be found in Montseny [2005]. We only mention that the space $\Delta_{\gamma}$ is an inductive limit of a family of Fréchet spaces $\widetilde{\boldsymbol{\Delta}}_{\gamma_{n}}$ isomorphic to $\boldsymbol{\Delta}_{\gamma_{n}}$ and based on a family of smooth contours $\gamma_{n}$ such that, in some sense, $\gamma_{n} \rightarrow \gamma$ (note that now, $\gamma$ is not necessarily smooth).

- Here again, the space $\Delta_{\gamma}^{\prime}$ is constituted of equivalence classes (of $\gamma$-symbols).

- Proposition 8 is still valid in the duality $\left\langle\Delta_{\gamma}^{\prime}, \Delta_{\gamma}\right\rangle$. 


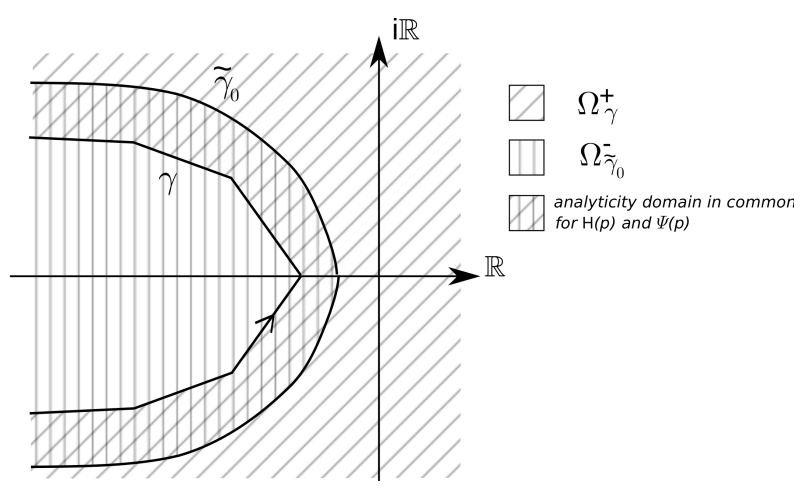

Fig. 2. Analyticity domains of $H$ and $\Psi$.

Canonical $\gamma$-symbols As $\gamma$ is non necessarily smooth, the canonical $\gamma$-symbol can not be defined as in $\boldsymbol{\Delta}_{\gamma}^{\prime}$.

Definition 6. For all $\mu \in \Delta_{\gamma}^{\prime}$, the canonical $\gamma$-symbol $\mu_{c}$ is defined by:

$$
\mu_{c}:=\lim \frac{\gamma_{n}^{\prime}}{2 \mathrm{i} \pi}\left[\mathcal{L} \mathcal{L}_{\gamma}^{*} \mu\right]\left(\gamma_{n}\right) \in \Delta_{\gamma}^{\prime},
$$

with $\gamma_{n}$ a sequence of smooth contours included in $\Omega_{\gamma}^{+}$ such that $\gamma_{n} \underset{n \rightarrow \infty}{\longrightarrow} \gamma$ in $W_{l o c}^{1, \infty}$.

Product $\sharp_{\gamma}$ in $\Delta_{\gamma}^{\prime} \quad$ Definition 5 of product $\sharp_{\gamma}$ is naturally extended to $\Delta_{\gamma}^{\prime}$. The product $\sharp_{\gamma}$ is inner in $\Delta_{\gamma}^{\prime}$ and it has been shown that product $\sharp_{\gamma}$ is sequentially continuous for the strong topology of $\Delta_{\gamma}^{\prime}$, that is:

$\left(\nu_{n} \underset{n \rightarrow \infty}{\stackrel{\Delta_{\gamma}^{\prime} \text { strong }}{\longrightarrow}} \nu, \mu_{n} \underset{n \rightarrow \infty}{\stackrel{\Delta_{\gamma}^{\prime}}{\underset{n}{\longrightarrow} \text { strong }}} \mu\right) \Longrightarrow\left(\mu_{n} \sharp_{\gamma} \nu_{n} \underset{n \rightarrow \infty}{\stackrel{\Delta_{\gamma}^{\prime} \text { strong }}{\longrightarrow}} \mu \sharp_{\gamma} \nu\right)$.

So, $\left(\Delta_{\gamma}^{\prime}, \sharp_{\gamma}\right)$ is a topological algebra (isomorphic to a commutative algebra of causal convolution operators). Note that $\Delta_{\gamma}^{\prime}$ is not unitary because the identity operator is not $\gamma$-diffusive.

\section{SUMMARY}

Let $\gamma$ be a closed simple arc in $\mathbb{C}^{-}$verifying the properties $(10,11)$. A $\gamma$-diffusive operator $H\left(\partial_{t}\right)$ admits the following state-realization:

$$
\begin{aligned}
\partial_{t} \psi(t, \xi) & =\gamma(\xi) \psi(t, \xi)+u(t), \psi(0, .)=0, \\
\left(H\left(\partial_{t}\right) u\right)(t) & =<\mu, \psi(t, .)>_{\Delta_{\gamma}^{\prime}, \Delta_{\gamma}},
\end{aligned}
$$

where $\mu \in \Delta_{\gamma}^{\prime}$ is the $\gamma$-symbol of $H\left(\partial_{t}\right)$. The bracket $<f, g>_{\Delta_{\gamma}^{\prime}, \Delta_{\gamma}}$ designates the extension (in the sense of the duality $\left\langle\Delta_{\gamma}^{\prime}, \Delta_{\gamma}>\right.$ ) of the scalar product $\int f g d \xi$. The essential conditions the operator has to satisfy to admit such a representation are:

- $H$ holomorphic in $\Omega_{\gamma}^{+}$,

- $H(p) \rightarrow 0$ when $|p| \rightarrow+\infty$ in $\Omega_{\gamma}^{+}$.

Thanks to the sector condition (10) verified by $\gamma$, the state realization is of diffusive type: so, cheap and precise numerical approximations of $(53,54)$ can be easily built.

\section{SOME EXTENSIONS}

\subsection{Operators $\gamma$-diffusive in the large sense}

Formulation $(53,54)$ can be extended to operators of the form $K\left(\partial_{t}\right) \circ \partial_{t}^{n}$ where $K\left(\partial_{t}\right)$ admits a $\gamma$-symbol in $\Delta_{\gamma}^{\prime}$; such operators are said $\gamma$-diffusive in the large sense of degree $n$. Formally, we have:

$$
\left[K\left(\partial_{t}\right) \circ \partial_{t}^{n} u\right](t)=<\mu, \partial_{t}^{n} \psi(t, .)>,
$$

with $\psi(t, \xi)$ solution of (53) and $\mu$ the $\gamma$-symbol of $K\left(\partial_{t}\right)$. In the particular case where $n=1,(57)$ can be written:

$$
\left[K\left(\partial_{t}\right) \circ \partial_{t} u\right](t)=<\mu, \gamma \psi(t, .)+u(t)>.
$$

In the same way, $\Delta_{\gamma}^{\prime}$ can be extended to an algebra denoted $\Sigma_{\gamma}$ whose elements are the $\gamma$-symbols of operators of the form $K\left(\partial_{t}\right) \circ \partial_{t}^{n}$ where $K\left(\partial_{t}\right)$ is associated to a $\gamma$-symbol $\mu \in \Delta_{\gamma}^{\prime}$ and $n \in \mathbb{N}$.

\subsection{General integral operators}

The above results can easily be extended to integral (non convolution) operators of the form $H\left(t, \partial_{t}\right)$, that is with kernel of the general form $\mathbf{h}(t, s)=h(t, t-s)$ such that $\forall t$, $H(t,)=.\mathcal{L} h(t,$.$) ; the \gamma$-symbol of $H\left(t, \partial_{t}\right)$ is then written as in the convolution case, by considering $t$ as a frozen parameter:

$$
\left(H\left(t, \partial_{t}\right) u\right)(t)=<\mu(t, .),\left(\Re \mathrm{d}_{\gamma} u\right)(t, .)>,
$$

where, for any $t, \mu(t,$.$) is the \gamma$-symbol of the convolution operator $H_{t}\left(\partial_{t}\right)$ with symbol $H(t,$.$) . Various other exten-$ sions will be found in (Montseny [2005]).

\subsection{Inversion of $\gamma$-symbols}

The inversion of $\gamma$-symbols cannot be defined in $\Delta_{\gamma}^{\prime}$ because this algebra is not unitary. This operation can nevertheless be well-defined in $\Sigma_{\gamma}$, under the condition that $0 \in \gamma$ that we can write without loss of generality $\gamma(0)=0$. In this case indeed, the Dirac distribution $\delta$ is a (non canonical!) $\gamma$-symbol of the integrator operator $\partial_{t}^{-1}$ and $\delta^{n}:=\delta \sharp_{\gamma} \delta \sharp_{\gamma} \ldots \sharp_{\gamma} \delta$ ( $n$ times) is a $\gamma$-symbol of $\partial_{t}^{-n}$. So, if $\mu \in \Sigma_{\gamma}$ is a $\gamma$-symbol of $K\left(\partial_{t}\right)$ such that $K\left(\partial_{t}\right)^{-1} \circ \partial_{t}^{-n}$ has a $\gamma$-symbol $\nu \in \Delta_{\gamma}^{\prime}$, then $\nu=\mu^{-1} \sharp_{\gamma} \delta^{n}$ and we have:

$$
\left[K\left(\partial_{t}\right)^{-1} u\right](t)=<\mu^{-1} \sharp \delta^{n}, \partial_{t}^{n} \psi(t, .)>,
$$

with $\psi$ solution of (53).

It has been shown in (Casenave [2009]) that the inversion operation is continuous in its definition domain for a suitably weakened topology.

\section{ABOUT NUMERICAL REALIZATIONS}

The state equation (53) is infinite dimensional. To get numerical approximations, we consider a sequence $\mathcal{M}_{L}$ of $L$ dimensional spaces of atomic measures on suitable meshes $\left\{\xi_{l}^{L}\right\}_{l=1: L}$ on the variable $\xi ; L$-dimensional approximations $\mu^{L}$ of the $\gamma$-symbol $\mu \in \Delta_{\gamma}^{\prime}$ are then defined in the sense of atomic measures, that is:

$$
\mu^{L}=\sum_{l=1}^{L} \mu_{l}^{L} \delta_{\xi_{l}^{L}}, \quad \mu_{l}^{L} \in \mathbb{C} .
$$

If $\cup_{L} \mathcal{M}_{L}$ is dense in the topological space $\Delta_{\gamma}^{\prime}$ (that is if $\cup_{L}\left\{\xi_{l}^{L}\right\}$ is dense in $\mathbb{R}$ ), then we have (Montseny [2005]):

$$
<\mu^{L}, \psi>\underset{L \rightarrow+\infty}{\longrightarrow}<\mu, \psi>\forall \psi \in \Delta_{\gamma}
$$


so, we have the following $L$-dimensional approximate state formulation of $H\left(\partial_{t}\right)$ (with $\gamma$-symbol $\mu$ ):

$\left\{\begin{array}{l}\partial_{t} \psi\left(t, \xi_{l}^{L}\right)=\gamma\left(\xi_{l}^{L}\right) \psi\left(t, \xi_{l}^{L}\right)+u(t), l=1: L, \psi\left(0, \xi_{l}^{L}\right)=0 \\ {\left[H\left(\partial_{t}\right) u\right](t) \simeq \sum_{l=1}^{L} \mu_{l}^{L} \psi\left(t, \xi_{l}^{L}\right),}\end{array}\right.$

and from (43):

$$
H(\mathrm{i} \omega) \simeq \sum_{l=1}^{L} \frac{\mu_{l}^{L}}{\mathrm{i} \omega-\gamma\left(\xi_{l}^{L}\right)} .
$$

Thanks to the sector hypothesis (10) on $\gamma$, most of non rational operators encountered in practice can be efficiently approximate with small $L$ (see for example Montseny [Nov. 2004]). Roughly speaking, this is a consequence of the property that damping is more and more efficient for high frequency components (associated with great values of $\xi$ ) of the impulse response of operator $H\left(\partial_{t}\right)$.

\section{VARIOUS EXAMPLES}

\subsection{First example}

We consider the operator $H\left(\partial_{t}\right)=\left(\partial_{t}+200\right)^{-1} \circ \ln \left(\partial_{t}\right)$ with Laplace-symbol:

$$
H(p)=\frac{\ln (p)}{p+200} .
$$

$H$ is holomorphic in $\mathbb{C} \backslash \mathbb{R}^{-}$and $H(p) \rightarrow 0$ when $|p| \rightarrow 0$ in $\mathbb{C} \backslash \mathbb{R}^{-}$; then $H\left(\partial_{t}\right)$ is $\gamma$-diffusive for $\gamma$ defined by $\gamma(\xi)=-|\xi|, \xi \in \mathbb{R}$. Its canonical $\gamma$-symbol, which is a distribution, is given by ${ }^{5}$ :

$$
\begin{aligned}
& \mu(\xi)=\operatorname{pv}\left(\frac{1}{|\xi|-200}\right)\left(\frac{1}{2}-\mathrm{i} \frac{\operatorname{sign}(\xi)}{2 \pi} \ln |\xi|\right) \\
& +\left(-\frac{1}{2}+\mathrm{i} \frac{\ln 200}{2 \pi}\right) \delta_{200}(\xi)+\left(-\frac{1}{2}-\mathrm{i} \frac{\ln 200}{2 \pi}\right) \delta_{-200}(\xi)
\end{aligned}
$$

and $H\left(\partial_{t}\right) u$ can be realized by $(53,54)$.

We consider a mesh $\left\{\xi_{l}^{L}\right\}_{l=1: L}$ of $L=70$ discretization points geometrically spaced between $\xi_{1}^{L}=10^{-3}$ and $\xi_{70}^{L}=$ $10^{4}$. To highlight the efficiency of the approximation, we show in figure 3 the Bode diagram of the exact Laplacesymbol of $H\left(\partial_{t}\right)$, the one obtained with formula (63), and the one given by $\frac{Y(\mathrm{i} \omega)}{U(\mathrm{i} \omega)}$ where $U=\mathcal{L} u$ and $Y=\mathcal{L} y$ with $u$ a white noise and $y$ the output obtained from numerical simulation of (62) in time domain. The approximation is very good in the frequency band $\left[10^{-3}, 10^{4}\right]$ covered by the $\operatorname{mesh}\left\{\xi_{l}^{L}\right\}_{l}$.

\subsection{Second example}

Now we consider the operator $H\left(\partial_{t}\right)=\partial_{t}^{-0.4} \circ\left(\partial_{t}+3\right)^{-1}$ with Laplace-symbol:

$$
H(p)=\frac{1}{p^{0.4}(p+3)} .
$$

$H$ is holomorphic in $\mathbb{C} \backslash \mathbb{R}^{-}$and $H(p) \rightarrow 0$ when $|p| \rightarrow 0$ in $\mathbb{C} \backslash \mathbb{R}^{-}$; so $H\left(\partial_{t}\right)$ is $\gamma$-diffusive for any $\gamma$ of the sector form:

$$
\left.\left.\gamma(\xi)=|\xi| \mathrm{e}^{\mathrm{i}\left(\frac{\pi}{2}+\alpha\right)} \text { with } \alpha \in\right] 0, \frac{\pi}{2}\right] .
$$

5 N.B.: pv $\frac{1}{\xi-a}$ classically designates the distribution associated with the non locally integrable function $\frac{1}{\xi-a}$ (it is derived from the Cauchy's principal value). The Dirac distribution at point $a$ is denoted $\delta_{a}$.
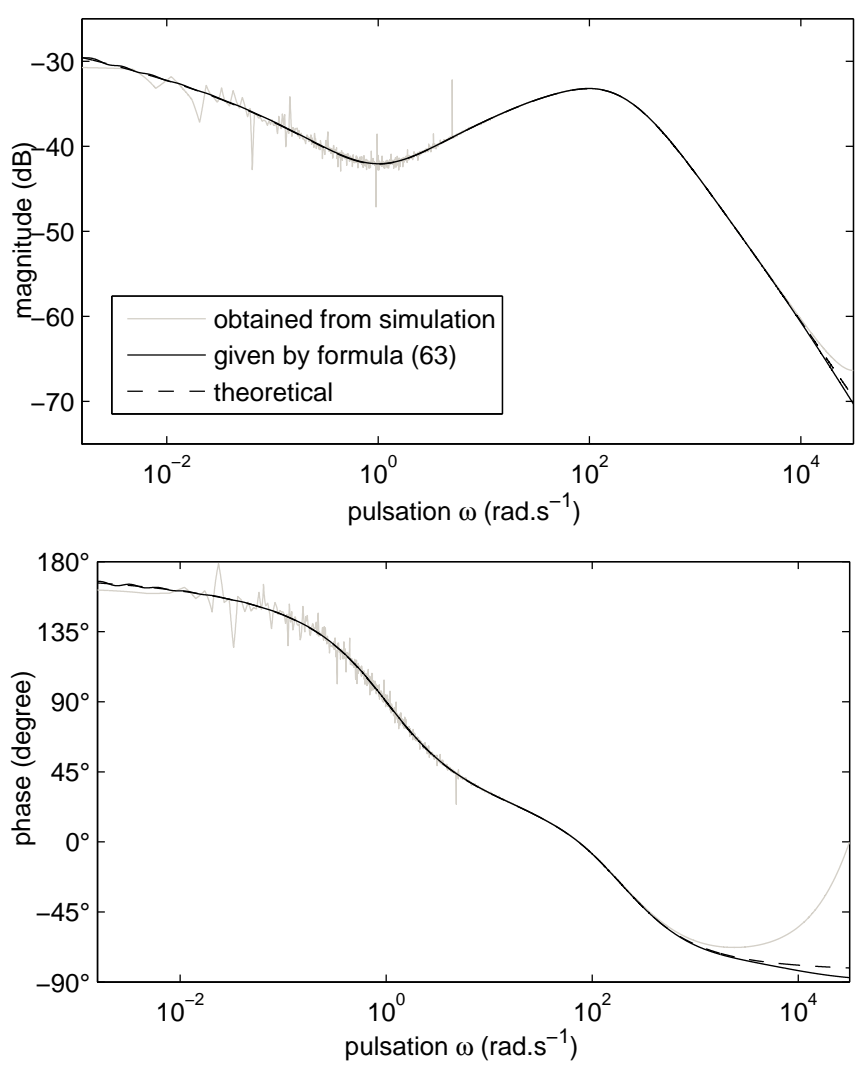

Fig. 3. Bode diagram of $\left(\partial_{t}+2\right)^{-1} \circ \ln \left(\partial_{t}\right)$

We consider here the contour $\gamma$ of the form (67) with $\alpha=$ $\frac{80 \pi}{180}$. Its canonical $\gamma$-symbol (which is a locally integrable function) is explicitly written:

$$
\mu(\xi)=\frac{\mathrm{e}^{\mathrm{i}\left(\frac{\pi}{2}+\alpha\right)} \operatorname{sign}(\xi)}{2 \mathrm{i} \pi|\xi|^{0.4} \mathrm{e}^{0.4 \mathrm{i}\left(\frac{\pi}{2}+\alpha\right)}\left(|\xi| \mathrm{e}^{\mathrm{i}\left(\frac{\pi}{2}+\alpha\right)}+3\right)} .
$$

We consider a mesh $\left\{\xi_{l}^{L}\right\}_{l=1: L}$ of $L=70$ discretization points geometrically spaced between $\xi_{1}^{L}=10^{-4}$ and $\xi_{70}^{L}=$ $10^{3}$. The approximation is shown in figure 4 . Here again the approximation is good on the frequency band $\left[10^{-3}, 10^{3}\right]$.

9.3 Other examples of $\gamma$-symbols in the case $\gamma(\xi)=-\xi$, $\xi \geqslant 0$

In the particular case $\gamma(\xi)=-|\xi|$ and with $h$ real impulse response, the symmetry with respect to $\xi=0$ can be used in order to restrict the $\gamma$-representation to $\xi \in \mathbb{R}^{+}$, that is:

$$
(\mathcal{H} u)(t)=\int_{0}^{+\infty} \mu^{+}(\xi) \psi(t, \xi) d \xi
$$

in such a case, the $\gamma$-symbol $\mu^{+}$is deduced from $H$ by the following expression (the limit must be understood in the sense of distributions) Montseny [2005]:

$$
\begin{aligned}
\mu^{+}(\xi) & =\frac{\mathbf{1}_{\xi>0}}{2 \mathrm{i} \pi}\left[H\left(\xi e^{-\mathrm{i} \pi^{-}}\right)-H\left(\xi e^{\mathrm{i} \pi^{-}}\right)\right] \\
& =\frac{\mathbf{1}_{\xi>0}}{2 \mathrm{i} \pi} \lim _{\varepsilon \rightarrow 0}[H(-\xi-\mathrm{i} \varepsilon)-H(-\xi+\mathrm{i} \varepsilon)] .
\end{aligned}
$$

Various $\gamma$-symbols $\mu^{+}$are given in table 1 . 

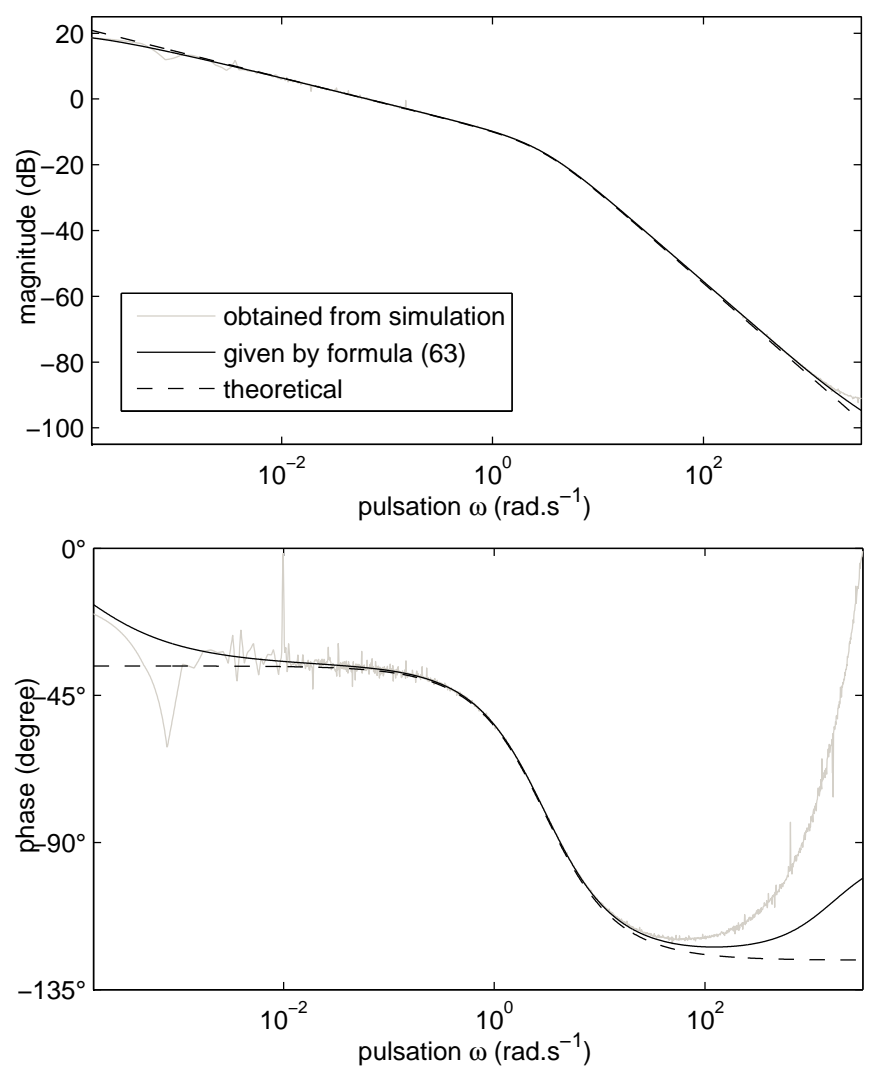

Fig. 4. Bode diagram of $\partial_{t}^{-0.4} \circ\left(\partial_{t}+3\right)^{-1}$

\begin{tabular}{|c|c|c|}
\hline conditions & $\begin{array}{l}\gamma \text {-symbol } \mu^{+} \\
(\xi>0)\end{array}$ & $\begin{array}{l}\text { Laplace-symbol } \\
H(p \in \mathbb{C})\end{array}$ \\
\hline \multirow[t]{2}{*}{$\mu d \xi=0$} & $-\xi \mu(t, \xi)$ & $p H(t, p)$ \\
\hline & $\partial_{\xi} \mu(t, \xi)^{(*)}$ & $-\partial_{p} H(t, p)$ \\
\hline \multirow{2}{*}{$a>0, b \geqslant 0$} & $\mu(t, a \xi-b)$ & $H(t, a p+b)$ \\
\hline & $\delta$ & $p^{-1}$ \\
\hline $\begin{array}{c}\operatorname{Re}(\alpha)>0 \\
\alpha \notin \mathbb{N}\end{array}$ & $\mu_{\alpha}(\xi):=\frac{\sin (\pi \alpha)}{\pi} \mathrm{fp} \frac{\mathbf{1}_{\xi>0}}{\xi^{\alpha}}$ & $p^{-\alpha}$ \\
\hline$\partial_{s}^{\alpha} h \in L_{\mathrm{loc}}^{1}$ & $\mu(t,.) \sharp \mu_{-\alpha}$ & $p^{\alpha} H(t, p)$ \\
\hline$a, b \geqslant 0$ & $\frac{1}{b-a} \delta_{a}+\frac{1}{a-b} \delta_{b}$ & $\frac{1}{(p+a)(p+b)}$ \\
\hline$a \geqslant 0, n \in \mathbb{N}^{*}$ & $\delta_{a}^{\sharp n}=\frac{1}{(n-1) !} \delta_{a}^{(n-1)}$ & $(p+a)^{-n}$ \\
\hline $\begin{array}{c}\operatorname{Re} \alpha>0 \\
a>0\end{array}$ & $\mu_{\alpha} \operatorname{pv} \frac{1}{(a-\xi)}+\frac{\cos (\pi \alpha)}{a^{\alpha}} \delta_{a}$ & $p^{-\alpha}(p+a)^{-1}$ \\
\hline $\begin{array}{c}-1<\operatorname{Re}(\alpha, \beta)<1 \\
\operatorname{Re}(\alpha+\beta)>0 \\
0<b<a\end{array}$ & $\begin{array}{l}\frac{\sin (\pi(\beta+\alpha)) \mathbf{1}_{\xi>a}}{\pi(\xi-a)^{\alpha}(\xi-b)^{\beta}}+ \\
\quad+\frac{\sin (\pi \beta) \mathbf{1}_{b<\xi<a}}{\pi(a-\xi)^{\alpha}(\xi-b)^{\beta}}\end{array}$ & $\frac{1}{(p+a)^{\alpha}(p+b)^{\beta}}$ \\
\hline$a>0$ & $\frac{1_{0<\xi<a}}{\pi \sqrt{\xi(a-\xi)}}$ & $\frac{1}{\sqrt{p} \sqrt{p+a}}$ \\
\hline $\begin{array}{c}-1<\operatorname{Re} \alpha<1 \\
0<b<a\end{array}$ & $\frac{\sin (\pi \alpha)}{\pi}\left(\frac{a-\xi}{\xi-b}\right)^{\alpha} \mathbf{1}_{b<\xi<a}$ & $\left(\frac{p+a}{p+b}\right)^{\alpha}-1$ \\
\hline $\begin{array}{c}0<\operatorname{Re} \alpha<1 \\
-\operatorname{Re} \alpha<\operatorname{Re} \beta \leqslant 1\end{array}$ & $\frac{\operatorname{Im}\left[\left(\xi^{\alpha} e^{i \pi \alpha}+a\right)^{\beta}\right]}{\pi\left(\xi^{2 \alpha}+2 a \cos (\pi \alpha) \xi^{\alpha}+a^{2}\right)^{\beta}}$ & $\left(p^{\alpha}+a\right)^{-\beta}$ \\
\hline \multirow[t]{6}{*}{$\gamma=$ Euler const. } & $-\mathrm{fp} \frac{\mathbf{1}_{\xi>0}}{\xi}$ & $-p^{-1}(\gamma+\ln p)$ \\
\hline & $\frac{1}{1+\xi}$ & $\frac{\ln (p)}{p-1}$ \\
\hline & $\frac{e^{-\bar{\xi}}}{\sqrt{\pi \xi}}$ & $\frac{\sqrt{\pi}}{\sqrt{p}} e^{p}(1-\operatorname{erf} \sqrt{p})$ \\
\hline & $\frac{\cos (\sqrt{\xi})}{\pi \sqrt{\xi}}$ & $\frac{\exp (-\sqrt{p})}{\sqrt{p}}$ \\
\hline & $\sum_{n \in \mathbb{Z}} \delta\left(\xi-n^{2} \pi^{2}\right)$ & $(\sqrt{p} \tanh \sqrt{p})^{-1}$ \\
\hline & $\sum_{n \in \mathbb{N}} \frac{a^{n}}{n !(2 n) !} \delta^{(2 n)} a$ & $\frac{1}{p} \exp \left(\frac{a}{p^{2}}\right)$ \\
\hline
\end{tabular}

(*) derivation in the sense of $\mathcal{D}^{\prime}(\mathbb{R})$

Table 1.
REFERENCES

Audounet, J., Giovangigli, V., and Roquejoffre, J.M. (1998). A threshold phenomenon in the propagation of a point-source initiated flame. Physica D, 121(3-4), $295-316$.

Audounet, J. and Roquejoffre, J. (1998). An asymptotic fractional differential model of spherical flame. European Series on Applied and Industrial Mathematics (ESAIM): Proceedings, 5(4), 15-28.

Bidan, P., Lebey, T., Montseny, G., Neacsu, C., and SaintMichel, J. (2001). Transient voltage distribution in motor windings fed by inverter: experimental study and modeling. IEEE Trans. on Power Electronics, 16(1).

Carmona, P., Coutin, L., and Montseny, G. (2003). Stochastic integration with respect to Fractional Brownian Motion. Annales de l'Institut H. Poincaré, 39(1), $27-68$.

Carmona, P. and Coutin, L. (1998a). Fractional brownian motion and the markov property. Electronic Communications in Probability, 3, 95-107.

Carmona, P. and Coutin, L. (1998b). Simultaneous approximation of a familiy of (stochastic) differential equations. European Series on Applied and Industrial Mathematics (ESAIM): Proceedings, 5.

Casenave, C. (2009). Représentation diffusive et inversion opératorielle pour l'analyse et la résolution de problèmes dynamiques non locaux. Ph.D. thesis, Université Paul Sabatier, Toulouse.

Casenave, C. and Montseny, E. (2008). Time-local dissipative formulation and stable numerical schemes for a class of integrodifferential wave equations. SIAM Journal on Applied Mathematics, 341, 1763-1783.

Casenave, C. and Montseny, G. (2009). Optimal Identification of Delay-Diffusive Operators and Application to the Acoustic Impedance of Absorbent Materials. In Topics in Time Delay Systems: Analysis, Algorithms and Control, volume 388, 315. Springer Verlag, Lecture Notes in Control and Information Science (LNCIS).

Degerli, Y., Lavernhe, F., and Magnan, P. (1999). Nonstationnary noise response of some fully differential onchip readout circuits suitable for CMOS image sensors. IEEE Trans on Circ. \& Syst. II, 46(12), 1461-1474.

Degerli, Y., Lavernhe, F., Magnan, P., and Farre, J. (Ap. 1999). Bandlimited $1 / f^{\alpha}$ noise source. Electronics Letters, 35(7), 521-522.

Devy-Vareta, F.A. and Montseny, G. (2001). Pseudoinvariant $\mathrm{H}_{2}$ multivariable robust control of a flexible beam. In European Control Conference ECC 2001. Porto (Portugal).

Garcia, G. and Bernussou, J. (1998). Identification of the dynamics of a lead acid battery by a diffusive model. European Series on Applied and Industrial Mathematics (ESAIM): Proceedings, 5, 87-98.

Helie, T. and Matignon, D. (2006). Diffusive reprentations for the analysis and simulation of flared acoustic pipes with visco-thermal losse. Mathematical Models and Methods in Applied Sciences, 16, 503-536.

Laudebat, L., Bidan, P., and Montseny, G. (2004). Modeling and Optimal Identification of Pseudodifferential Electrical Dynamics by Means of Diffusive Representation - Part I : modeling. IEEE Trans. on Circ. \& Syst. $I, 51(9)$. 
Lavernhe, F., Montseny, G., and Audounet, J. (2001). Markovian diffusive representation of $1 / f^{\alpha}$ noises and application to fractional stochastic differential models. IEEE Trans. on Signal Processing, 49(2).

Lavernhe, F. and Solhusvik, J. (1998). Fractional noises: diffusive model for CCD imager acquisition chain. ESAIM: Proc., 5.

Leger, S. and Pontier, M. (1999). Drap Brownien fractionnaire. Comptes Rendus de l'Académie des Sciences, Paris, série I, , 329, 893-898.

Lenczner, M. and Montseny, G. (2005). Diffusive realization of operator solutions of certain operational partial differential equations. CRAS - Mathématiques, 341(12), $737-740$.

Lenczner, M., Montseny, G., and Yakoubi, Y. (2010). Diffusive realizations for solution of some operatorial equation: the one-dimensional case. to appear in Mathematics of Computation.

Levadoux, D. and Montseny, G. (2003). Diffusive formulation of the impedance operator on circular boundary for 2D wave equation. In The Sixth International Conference on Mathematical and Numerical Aspects of Wave Propagation. Jyväskylä (Finland).

Lubich, C. and Schädler, A. (2002). Fast convolution for nonreflecting boundary conditions. SIAM J. Sci. Comput., 24(1), 161-182.

Matignon, D. (July 2001). Damping models for mechanical systems using diffusive representation of pseudodifferential operators: theory and examples. In Proc. workshop on pluralism in distributed parameter systems, 88-90. Enschede, The Netherlands.

Matignon, D. and Prieur, C. (2005). Asymptotic stability of linear conservative systems when coupled with diffusive systems. ESAIM: Control, Optimisation and Calculus of Variations, 11(3).

Mbodge, B. and Montseny, G. (1995). Boundary fractional derivative control of the wave equation. IEEE Trans. on Aut. Cont., 40(2), 378-382.

Mbodge, B., Montseny, G., and Audounet, J. (1994). Analysis of fractionally damped flexible systems via a diffusion equation. International Journal of Systems Science, 25(11), 1775-1791.

Montseny, G. (1991). Diffusion monodimensionnelle et intégration d'ordre 1/2. Internal LAAS Report $N$. 91232.

Montseny, G. (1998). Diffusive representation of pseudodifferential time-operators. European Series on Applied and Industrial Mathematics (ESAIM): Proceedings, 5, $159-175$.

Montseny, G. (2002a). Contrôle diffusif pseudo-invariant: principes et exemples. In Avancées récentes en commande robuste - Applications à la mécanique des structures. Ecoles CEA-EDF-INRIA Problèmes Non Linéaires Appliqués, INRIA - Rocquencourt, France.

Montseny, G. (2002b). Diffusive representation: a new concept for complex dynamic problems involving pseudodifferential operators. In Lecture notes of the Summer School: On the links between nonlinear physics and information sciences.

Montseny, G. (2005). Représentation diffusive. Hermes Science Publ.

Montseny, G. (2007). Diffusive representation for operators involving delays. In Applications of Time-delay
Systems, 217-232. Springer Verlag, Lecture Notes in Control and Information Science (LNCIS).

Montseny, G. (Nov. 2004). Simple approach to approximation and dynamical realization of pseudodifferential time-operators such as fractional ones. IEEE Trans on Circuits and System II, 51(11), 613-618.

Montseny, G., Audounet, J., and Matignon, D. (2000). Diffusive representation for pseudodifferentially damped nonlinear systems. Nonlinear control in the year 2000 , $2,163-182$.

Montseny, G., Audounet, J., and Mbodge, B. (1993a). Modèle simple d'amortisseur viscoélastique. Application à une corde vibrante. Lecture Notes in Control and Information Sciences, Eds. RF.Curtain, A.Bensoussan, JL.Lions - Springer Verlag, 185, 436-446.

Montseny, G., Audounet, J., and Mbodge, B. (1993b). Optimal models of fractional integrators and application to systems with fading memory. In IEEE International Conference on Systems, Man and Cybernetics, Le Touquet (France), 17-20 Octobre 1993, 65-70.

Mouyon, P. and Imbert, N. (2002). Identification of a 2D turbulent wind spectrum. In Aerospace Science and Technology, volume 6, 3599-605. Kansas City (USA).

Nihtila, M. and Tervo, J. (2002). Pseudo-differential boundary control problems and applications to dynamics and control of bioprocesses. In MaDaMe Conference, Haikko Manor. Porvoo (Finland).

Rouzaud, H. (1998). Dynamique d'un modèle intégrodifférentiel de flamme sphérique avec pertes de chaleur. Comptes Rendus de l'Académie des Sciences, série I, 327.

Rumeau, A., Bidan, P., Lebey, T., Marchin, L., Barbier, B., and Guillemet, S. (2006). Behavior modeling of a $\mathrm{cacu}_{3} \mathrm{ti}_{4} \mathrm{O}_{12}$ ceramic for capacitor applications. In IEEE Conference on Electrical Insulation and Dielectric Phenomena. Kansas City (Missouri USA).

Staffans, O. (1994). Well-posedness and stablizability of a viscoelastic equation in energy space. Transactions of the American Mathematical Society, 345, 527-575.

Yosida, K. (1965). Functional analysis. Springer-Verlag, Berlin. 